\title{
La programación televisiva mexicana de 1999 desde la perspectiva de la diversidad ${ }^{1}$
}

José Carlos Lozano Rendón

Juan Enrique Huerta Wong ${ }^{2}$

¿CÓMO SE MIDE LA CALIDAD de los sistemas televisivos actuales? ¿Tenemos una mejor televisión que en décadas anteriores? ¿Han sido positivos para el televidente los cambios ocurridos en los últimos veinte años en términos de privatización, desregulación y liberalización de los medios electrónicos en la mayoría de los países del mundo?

Preguntas como las anteriores resultan fundamentales hoy en día, cuando la televisión sigue consolidándose como la principal opción de millones de personas para informarse, entretenerse y educarse informalmente y cuando los sistemas de televisión de paga por cable o por satélite se han expandido dramáticamente. Irónicamente, a la par con este acrecentamiento de la importancia de los medios electrónicos en la vida política, económica, social y cultural de los ciudadanos, los gobiernos han tendido a adoptar políticas de desregularización, mostrando menos interés en controlar o vigilar a los medios y sus contenidos desde la perspectiva del interés público (cfr. Golding, 1998).

El caso mexicano, pese a sus diferencias marcadas con el europeo y el norteamericano, participa de alguna manera de esta tendencia mundial. En Europa occidental, la desregulación rompió el monopolio de la televisión pública estatal y creó sistemas mixtos donde las opciones comerciales pasaron a ser más fuertes e importantes (cfr. De Bens, 1998; Gutiérrez, 2000). Más que impulsar la deseada diversidad de contenidos, estos cambios han propiciado una preocupante convergencia hacia "más canales, más de lo mismo", al grado de que algunos autores europeos hablan de la generación de tres paradojas, donde el explosivo crecimiento de canales en los sistemas de paga irónicamente no ha propiciado ni mayor diversidad, ni mayor información ni mayor comunicación (cfr. Cuilenberg, 1998: 44).

1. Este es un trabajo del Programa de investigación Televisión y Vida Cotidiana, que dirige José Carlos Lozano Rendón en el Tecnológico de Monterrey, con apoyo de la Cátedra Televisa.

2. ITESM, campus Monterrey. 
En México, después de la fusión en 1973 de Telesistema Mexicano y Televisión Independiente de México, Televisa dominó el mercado televisivo comercial mexicano hasta 1993, cuando la administración de Carlos Salinas de Gortari privatizó los canales estatales de IMEVISIÓN (fundados en 1983) dando pie al surgimiento de Televisión Azteca. En consonancia con las políticas neoliberales en boga en los últimos años en toda América Latina, la consigna fue desprenderse de empresas estatales y entregarlas a la iniciativa privada. Con el surgimiento de la nueva cadena, los defensores de la privatización auguraron mayor oferta y mayor diversidad de contenidos y opiniones. La competencia beneficiaría principalmente al público, pues habría otro tipo de programas y se proporcionaría acceso a otro tipo de actores sociales.

A siete años de ese cambio radical en el sistema televisivo mexicano, vale la pena realizar un diagnóstico que permita evaluar el grado de diversidad y de servicio público que están ofreciendo ambas cadenas al auditorio televisivo nacional. Mediante un análisis de la programación de cuatro semanas de 1999 (una para cada trimestre del año) de los cuatro principales canales nacionales de ambas empresas (Canal 2, Canal 5, Canal 7 y Canal 13), el presente trabajo ofrece evidencias sobre la diversidad que mostró la televisión abierta mexicana durante ese año, mediante la medición de la procedencia y el género de sus contenidos.

\section{Televisión e interés público}

Los medios de comunicación constituyen el nuevo espacio de lo público, por lo que es preciso cuestionarse cuál es su responsabilidad en la construcción del interés público, es decir, si sirven a los fines de toda la ciudadanía en lugar de servir solamente a los de cierto sector (cfr. McQuail, 1998).

Los principales elementos del interés público según la Federal Communications Commission (FCC) de Estados Unidos son el equilibrio, la heterogeneidad, el dinamismo, el localismo y la diversidad (p. 89). Es deseable que los medios de comunicación masiva se fundamenten en principios como libertad de expresión y de información, lo cual significa que cualquiera tiene derecho a expresar su opinión, así como informar y ser informado del mundo que le rodea. Deben existir también condiciones estructurales (normatividad), condiciones de funcionamiento (independencia real frente a presiones económicas y políticas, tanto a nivel organizacional como personal), acceso y calidad según criterios de diversidad, confiabilidad, interés, originalidad y satisfacción personal.

Desgraciadamente, hay pocos acuerdos en la discusión respecto a qué formas en la estructura -propiedad- de los medios garantizan esos principios en los procesos de emisión, oferta, contenidos y consumo de medios de comunicación. Frecuentemente se ha creído que poner a los medios en manos de la sociedad era un 
buen comienzo, y se consideró que la iniciativa privada representaría a la sociedad. Sin embargo, como señala Thompson (1998), el exceso de la aplicación de este razonamiento ha derivado en la formación de monopolios que limitan el acceso a todos, por lo menos a todos los que no pueden, directa o indirectamente, pagar por ello. Las empresas "se guían por el beneficio y acumulación de capital, y no existe necesariamente una correlación entre la lógica del beneficio y el cultivo de la diversidad" (pp. 308-309).

El interés público, agrega McQuail, no se puede basar solamente en el libre mercado ni en medios estatales. La calidad de información y de los contenidos cuiturales que recae en la responsabilidad social de los medios no puede ser vista desde el criterio del mercado. Y la responsabilidad de los medios de comunicación consiste en participar en la construcción del interés público, es decir, velar por la participación de todos los actores sociales. Servir a ciudadanos antes que a clientes.

Entre los elementos que deben intervenir está presente la lógica de la diversidad como el derecho a tener opciones y el compromiso de reconocer y respetar las diferencias entre individuos y/o colectividades (Ishikawa y Muramatzu, 1996).

Para fines de este trabajo, desde la visión del interés público, se entenderá a la calidad como diversidad. Una definición de la diversidad es "la variabilidad massmediática (fuentes, canales, mensajes y audiencias) de acuerdo con las diferencias relevantes en la sociedad (políticas, geográficas, socioculturales, etc.)" (McQuail, 1998 : 221).

El objetivo central de la diversidad es "que los receptores de emisiones tengan derecho a 'recibir un acceso adecuado para sus ideas y experiencias sociales, políticas, estéticas, morales y de otro tipo"' de acuerdo con la Comisión Federal de Comunicaciones de Estados Unidos de América -en adelante FCC- (McQuail, 1998: 214-215). Es un medio y un fin en sí mismo. "En una sociedad pluralista, todos los grupos deben tener los mismos derechos, si no el mismo status" (p. 216).

McQuail ha dicho que existen también dos principios de diversidad: puede ser dividida en diversidad como acceso y como oferta... [la primera] ofrece oportunidades para más o menos emisores [...] para ejercer su competencia profesional, pese a que ellos frecuentemente se adhieren a varias escuelas de pensamiento. También ofrece oportunidades para satisfacer los intereses de varios otros grupos de receptores -'el gran público'- [...] finalmente, la diversidad en programación (diversidad como oferta) es también un medio para garantizar un alto nivel de calidad descriptiva acumulativa, desde que los varios tipos de programas ofrecen descripciones diferentes, interpretaciones y explicaciones de la [...] realidad. La diversidad [...] entonces se convierte en un valor central por sí misma (en Hillve, Majanen y Rosengren: 294-295, cursivas en el original).

Hillve (1995), agrega a estos dos principios un tercero, que denomina oportunidad (que McQuail introduce como condición al principio de acceso), de los cuales derivan dos aspectos, denominados diversidad cultural -que puede ser medido 
por la distribución de producción doméstica y extranjera- y diversidad de tipos, que puede ser definida como "una medida de la probabilidad que un horario de programa satisfará la necesidad de variedad de un espectador individual [...] a más tipos ofrecidos, más grande la libertad de oportunidad y más grande la libertad de oportunidad de la audiencia" (p. 27, cursivas propias).

McQuail (1998) sugiere que un ambiente ideal en beneficio del interés público es promover la diversidad alentando distintos tipos de estructura de medios, que el sistema promueva el acceso (p. e. en materia electoral) y cada canal el reflejo (p. 221). Así llegamos a las definiciones de diversidad horizontal y vertical.

La diversidad horizontal se refiere al número de programas o tipos de programas diferentes disponibles para el espectador/oyente en un momento determinado. La diversidad vertical mide el número de programas (o tipos de programas) diferentes que ofrece un canal (o grupo de canales) a lo largo de toda la programación (de una noche, una semana, un mes, etc.). (p. 225)

Considerando estos cuatro puntos de vista acerca de la diversidad, McQuail (1998) propone que la diversidad puede evaluarse

[...] en el nivel de la estructura (externa/interna), o el espectro de opciones en los canales; en el nivel de la acción (criterio de igualdad o proporcionalidad); en relación con la cantidad y con la clase de la representación y el acceso; por referencia a la dimensión horizontal o vertical del tiempo (p. 225, cursivas en el original).

Es decir, habría que revisar qué tanta diversidad existe en el sistema de canales, en la programación y contenidos de la oferta de la televisión, en un canal y horario establecido; asimismo, observar la relación entre la cantidad de la producción y la diversidad de la misma.

Como antes se dijo, existen dos aspectos a considerar en la evaluación de la programación: diversidad de tipos y cultural. La primera mide cuántos tipos distintos de programas existen y se entiende que habrá una mayor libertad de oportunidad de la audiencia si hay un mayor número de tipos ofrecidos (Hillve, 1995: 27). La segunda deriva de la primera y atiende a la preocupación que algunas naciones han mostrado respecto a la definición de identidades culturales (Raboy, 1996a). La investigación en comunicación rechazó hace mucho tiempo la existencia de efectos directos de los medios en sus audiencias y propuso que existen, por un lado, mediaciones que propician una apropiación diferenciada de los medios, y por otro, mecanismos de negociación de los impactos en los habitus de las audiencias. Sin embargo, también existe evidencia respecto a cierto grado de influencia de la televisión en los públicos, razón por la que países como Canadá (De la Garde, 1993), Dinamarca (Sondergaard en Hultén, 1995), o Nueva Zelanda (Norris y Pauling, 1999), han mostrado preocupación por el flujo de programas extranjeros en sus televisiones. 


\section{Diversidad en la programación, dependencia en la estructura}

En distintas partes del mundo, los investigadores han realizado análisis de las programaciones televisivas para evaluar el grado de diversidad que se presenta en sus contenidos. Kim (1996), por ejemplo, comparó a los sistemas televisivos de Estados Unidos, México (tradición televisiva comercial); Inglaterra, Francia (tradición televisiva pública); Corea y Japón (tradición televisiva mixta). Utilizando el análisis de regresión como medida, sólo encontró, en materia de formatos, diferencias estadísticas en materia de entretenimiento, cuya frecuencia es mucho mayor en tanto hay más presencia de canales comerciales. Encontró asimismo evidencia suficiente para respaldar las hipótesis de que: a) un país con más canales de televisión tendrá más alta diversidad horizontal de programas, b) los canales públicos tienen mayor grado de diversidad que los comerciales y c) la diversidad es mayor en los sistemas abiertos que privados.

En el eje de las preocupaciones de los emisores por seguir siempre la forma establecida de conseguir el mayor número de audiencia, está el argumento de la factibilidad económica. Como hemos dicho, Levin (1971) encontró que las empresas multimedia ofrecen el menor grado de diversidad. Pero hoy, en los albores del siglo XXI, con los grandes monopolios ejerciendo el-control de la mayoría de formas simbólicas transmitidas masivamente en el mundo (Roach, 1997; Thompson, 1993), se debe cuestionar si la diversidad puede ser factible para grupos en desventaja económica, particularmente en un sistema de televisión tradicionalmente comercial como el mexicano.

\section{Diversidad cultural}

En el centro de las preocupaciones en investigación de la diversidad ha estado el tema de la diversidad cultural. Para algunos países, como Canadá (Raboy, 1996a) o Nueva Zelanda (Norris y Pauling, 1999), la diversidad cultural ha sido la categoría importante al definir la calidad.

La constante es la concentración de las importaciones de programación estadounidense. Raboy (1996b) encontró en la televisión canadiense $30.8 \%$ de importaciones estadounidenses, de los que 55.6\% son películas y series. En combinación con las cifras de importaciones inglesas en materia educativa, argumenta que existe una cierta colonización de los públicos franceses por las producciones en idioma inglés, lo que le parece preocupante. Si en materia de diversidad de tipos la televisión canadiense ya puede encontrar razones para preocuparse (43\% es información/intereses personales/educación y $57 \%$ es entretenimiento/ficción, que se dis- 
para a más del 75 en horario triple A), el hecho de que ellos definan su diversidad programática desde el punto de vista cultural encuentra motivos de preocupación en lo que parece ser un ascenso en las importaciones estadounidenses, particularmente de ficción (cfr. De la Garde, 1993; Gates, 1998; Raboy 1996b; Tremblay y Gauvreau, 1995).

En Taiwán, a partir de una privatización reciente de las comunicaciones, las importaciones se han disparado. Las provenientes de Estados Unidos llegaron a ocupar el 99\% de su programación, apuntan Ischii, Su y Watanabe (1999), quienes reportan una división del trabajo en la ficción importada, en la que la japonesa ocupa un muy alto rango de importaciones destinado a público muy joven o muy adulto, como caricaturas y series clásicas, en tanto que las importaciones estadounidenses, también muy altas, son líderes en los programas destinados al público medio.

Tres factores parecen influir en países con alto número de importaciones. Por un lado, está la afinidad geolingüística, mencionada entre otros por Sánchez Ruiz (1996); por otro, la vecindad (De la Garde, 1993); otro más sería una baja producción de programación local, que facilite el acceso de programación. En el primer caso podemos mencionar a países como Canadá (De la Garde, 1993); en el segundo a Dinamarca (Lerche-Nielsen y Wechselmann, 1993); y en el tercero a Sudáfrica y Nueva Zelanda (Norris y Pauling, 1999). Aunque es natural que estos factores puedan estar concatenados. Conviene recordar, como antes se dijo, que pese a haber una alta cantidad de importaciones en materia de ficción en el caso estadounidense, y en educación en el inglés, el flujo no es unidireccional, como habría sugerido Varis (1988) hace ya algunos años, sino que está sujeto a un flujo denominado interdependencia asimétrica (Sánchez Ruiz, 1996), aunque estudiar a fondo este fenómeno no es objetivo de este trabajo, sino observar cuál es el grado de diversidad en la programación nacional abierta.

Es preciso comentar sin embargo, que es frecuente que la programación local sea mayor a la extranjera (cfr. Biltereyst, 1992), lo cual no es razón para hacer a un lado este tipo de preocupaciones. Como hemos visto a partir de los datos de Norris y Pauling (1999), hay que tomar en cuenta que existe un cierto dominio en el flujo de contenidos de ficción, particularmente el proveniente de Estados Unidos.

También es preciso reiterar que el hecho de que exista una programación preferente, no significa que exista siempre un consumo preferente de esta programación.

\section{El caso de México}

La preocupación en México, probablemente producto del perfil ideológico que frecuentemente ha tenido la investigación en Latinoamérica, se ha enfocado más a 
los posibles efectos de la televisión extranjera, particularmente estadounidense, en las audiencias.

En principio, se pueden mencionar las que Lozano (1995/96) llama condiciones necesarias para conocer la dimensión de los efectos en las audiencias, que son conocer la oferta de los medios, el tipo de mensajes que reproducen y promueven, la exposición a estos contenidos y la apropiación de dichos mensajes por las audiencias. Como ya hemos dicho, este trabajo se enfoca únicamente a la primera parte del proceso de comunicación masiva, es decir, al análisis de la oferta. Aunque Lozano se refiere a mensajes transnacionales, el modelo parece extensible a los fines que aquí se exponen, como de hecho lo hace este autor en otro trabajo (Lozano y García Núñez de Cáceres, 1995).

También Lozano (1997) es quien menciona que respecto al primer punto (el de los estudios de la oferta) parece haber un relativo auge en nuestro país durante los últimos años. Esto en contraste con años anteriores en que el problema pasó inadvertido a los investigadores mexicanos, según se desprende de una revisión bibliométrica hecha a principios de la década (cfr. Sánchez Ruiz, 1992).

Aceves (1991) argumenta que existen dos grandes tendencias en el estudio de la televisión en México: el tema de la diversidad y el tema de televisión y niños. En realidad, sin embargo, los estudios sistemáticos en el primer renglón fueron prácticamente inexistentes por mucho tiempo, como se puede ver en Fuentes Navarro $(1996,1998)$ y en el ya citado Sánchez Ruiz (1992). No fue sino hasta el esfuerzo que con motivo de la discusión de políticas culturales en el contexto de la firma del Tratado de Libre Comercio con América del Norte -TLCAN-, hicieron académicos de México y Canadá (Tremblay y Gauvreau, 1995; Crovi Druetta y Vilar Alcalde, 1995; Lozano y García Núñez de Cáceres, 1995; Sánchez Ruiz, 1995), cuando se efectuaron estudios más representativos y amplios que concluyeron que en la televisión canadiense y mexicana existía una marcada concentración en torno al entretenimiento como formato de programación, lo cual destacaba aún más en horario Triple A.

En el aspecto de la diversidad cultural, se puede observar que las importaciones procedían casi exclusivamente de Estados Unidos. En México esto parece ser más regla que excepción, de acuerdo con el meta-análisis de la investigación de flujos de programación de Lozano (1997, julio), quien muestra que los formatos ficción e información conforman frecuentemente más del 50 por ciento de la oferta televisiva, con un sobrado énfasis del primero en las horas de mayor audiencia (cuadros 1 y 2).

En un estudio mayor y más reciente, Lozano (1998) estudió la oferta televisiva disponible en Monterrey desde este mismo enfoque. En la televisión abierta, destacaba la mayor presencia de contenido de entretenimiento estadounidense sobre el resto de formatos, de manera más visible en el tipo películas $(1,032.5$ horas de películas estadounidenses en 1,973 horas totales en la muestra). El único formato 
diferente que presentaba algún grado de concentración, los noticieros, no tenía mayor peso en el horario AAA. Este fenómeno también se presentaba en los canales privados, la mayoría en la muestra (41 de 47).

Kim (1996), que como dijimos, analizó la oferta de los sistemas televisivos en Japón, México, Francia, Corea, Reino Unido y Estados Unidos, encontró que sólo el sistema televisivo japonés tuvo una menor diversidad horizontal que el mexicano y la comparación le sirvió para apoyar su hipótesis de que un país con más canales de televisión tenderá a tener mayor diversidad en la programación, la cual, como apuntamos antes, es contrapunteada por Hultén (1995) e Ishikawa et al. (1996), quienes sugieren que no sólo es desaparecer los monopolios de una sola empresa, sino del sector privado en general.

En cualquier caso, es preciso cuestionarnos a partir de los datos de Kim, cuál es el rol que juega la estructura del sistema televisivo en su oferta programática.

Este trabajo pretende arrojar evidencia de la diversidad que mostró la televisión abierta mexicana durante 1999 y forma parte del Programa de Investigación sobre Televisión y Vida Cotidiana de la Cátedra Televisa que dirigió José Carlos Lozano Rendón en el Tecnológico de Monterrey en ese año. Se mide principalmente la diversidad de orígenes y tipos en que se presenta la programación transmitida por la televisión abierta mexicana, como un primer paso a la discusión por lograr medios de comunicación de calidad en la transición a la democracia.

En este sentido, son recuperadas dos preguntas de investigación planteadas por Lozano y García Núñez de Cáceres en 1995:

¿Cuáles son los tipos televisivos más difundidos y qué tan equilibrada se encuentra la oferta de los mismos?

¿Qué tan amplia es la oferta de programación extranjera en los canales [nacionales de televisión abierta mexicana]..., tanto en horario general como en el AAA?

\section{Metodología ${ }^{3}$}

La muestra se compuso de cuatro semanas de programación. El criterio fue seleccionar la segunda semana del segundo mes de cada trimestre. Se dividió el periodo de análisis (un año) en cuatro partes, y se escogieron las semanas justo en medio de cada trimestre, con el fin de eliminar sesgos. El primer instrumento de trabajo fue la página de cartelera del periódico de circulación nacional Excélsior, publicada en su sección nacional, de donde se codificó la información. No se realizó muestreo

3. Este trabajo se basa en el Proyecto Monitoreo y diagnóstico de la oferta televisiva, que coordinó Aída Cerda Cristerna como parte del equipo de trabajo del Programa Televisión y Vida Cotidiana. Este programa consta de 10 proyectos de investigación y diseminación de investigación acerca de la oferta y recepción de la televisión mexicana. 
de unidades de análisis, sino que se censaron ambas semanas, codificando toda la programación de los canales que conforman el estudio. Se aplicaron pruebas piloto y de confiabilidad a la programación de la semana 12-18 de abril, contando con un manual y hoja de codificación de variables semiabiertas, tras lo cual se despejaron dudas acerca de los subgéneros programáticos y se cerraron las variables hasta hacerlas nominales, para integrar un manual y hoja de codificación finales.

La unidad de análisis fue el programa y para fines operacionales, se codificó el número de minutos que cada uno registró, de modo que el análisis está hecho a partir de los minutos de programación y no del número de programas que conforman la muestra. Las variables principales entonces fueron: canal, minutos, formatos y tipos (tabla 1), país de origen y horario de programación.

\section{Resultados}

La evidencia tendió a mostrar que la diversidad escaseó en la oferta de la televisión abierta mexicana durante 1999. La ficción fue la que más predominó (28\%), sobre todo en el horario Triple A. La excepción fue la última semana, que tuvo una mayor inclinación a los programas infantiles ${ }^{4}$ (Cuadro 2).

Es importante observar en el Cuadro 2, que tres formatos (ficción, infantiles e información) mantienen una concentración que oscila de 69 -en el caso de la cuarta semana- a 80 por ciento en el caso de la segunda. Salvo la cuarta semana de muestra, que es en general la que obtuvo los resultados menos concentrados, incluso los dos formatos dominantes, ficción e infantiles, ostentan más del 50 por ciento de la programación total, lo cual se da incluso como media (53\%) de todo el año. En general podemos señalar que los formatos televisivos transmitidos por televisión abierta en México durante 1999, mostraron alta concentración.

Los datos del Cuadro 2 parecen sugerir que el público infantil puede estar recibiendo una oferta altamente concentrada de un sólo formato programático y, como se muestra en el desglose de tipos, ${ }^{5}$ particularmente en el tipo de dibujos animados. Esto hace pertinente preocuparse por las probables visiones de realidad que niños y adolescentes en etapas de formación intelectual pudieran percibir

4. Aquí se observan problemas con respecto a la tipología planteada por el sistema televisivo mexicano, como han apuntado algunos críticos (Kim, 1996; Litman y Hasegawa, 1996). El formato "infantiles" en realidad representa un público y no un tipo de formato, como critican Litman y Hasegawa en su revisión de la literatura en Estados Unidos de América. Se puede señalar también que el formato infantiles incluye dibujos animados o series infantiles, que formarían parte del formato de ficción; como éste es un primer esfuerzo en este orden de trabajos, valía la pena por ahora abordar la problemática desde la nomenclatura del sistema televisivo, pero es un dato que nos puede servir para abandonar esta práctica en estudios posteriores.

5. Por motivos de espacio, los desgloses por tipos de todas las variables fueron suprimidos. Se pueden confrontar en Huerta Wong (2000a). 
desde una televisión que muestra sólo una particular forma de ver el mundo, pues en principio varios tipos de programas pueden ofrecer descripciones, interpretaciones y explicaciones de la realidad distintas, como ha dicho McQuail (en Hillve, Majanen y Rosengren, 1997: 295). Además, esto nos devuelve al plano de las preocupaciones por los efectos, que origina gran parte de estudios en el área de comunicación de masas, si bien es preciso insistir en que es sólo una llamada de atención pues hacen falta análisis de otro tipo para dar cuenta exacta de los mensajes transmitidos por la televisión.

Si la oferta programática general del sistema de canales de la televisión abierta nacional fue poco diversa, es momento de ver qué ocurrió en cada canal y horario. Los datos siguientes hablan del conjunto y no de cada periodo de la muestra, dado que a pesar de las diferencias aparentes entre las semanas, más notorias en el caso del formato ficción, el objetivo de este trabajo es dar una visión general sobre la oferta televisiva global durante el año de 1999.

El Cuadro 3 nos introduce al tema de la diversidad vertical. En casi todos los casos sólo dos formatos ocupan más del 50 por ciento de la muestra, salvo el canal 7 de TV Azteca, que tendió a mostrar una menor concentración. Como se dijo, los tres formatos centrales son información (21\%), ficción (28\%) e infantiles (24\%). Parece haber una división del trabajo, donde los canales 2 en el caso de Televisa y 13 de TV Azteca, se ocupan mayoritariamente de la ficción (31\% en ambos casos) y la información ( 36 y $38 \%$, respectivamente), y los canales 5 de Televisa y 7 de TV Azteca, de la programación infantil ( 67 y $27 \%$, respectivamente). El importante peso del canal 7 de TV Azteca en la ficción explica el desequilibrio en favor de este formato como predominante en la oferta programática abierta mexicana. Pero si el canal de menor concentración es el 7 de TV Azteca, esta empresa se ocupa también del canal 13, el de mayor concentración (sólo dos formatos ocupan $71 \%$ de su programación), que parece un canal especializado. La diversidad vertical es baja en todos los casos. Al descomponer estos datos en tipos, observamos que en realidad el grado de concentración es mayor en los canales 2 y 5 de Televisa que en los de TV Azteca, aunque estos datos no son incluidos aquí, como se ha explicado.

Respecto a la diversidad horizontal, el Cuadro 4 muestra que ésta es baja en general, pero que existe un giro en la programación de los formatos en diferentes horarios. En tanto que hay un abultamiento de la programación infantil en los horarios A (30\%) y Doble A (33\%), esto disminuye en el horario Triple A. ${ }^{6}$ En este horario, la ficción registra una mayor concentración (51\%). Este formato muestra a lo largo del día una tendencia considerable a la alza. Con algunas variaciones más o menos obvias en cada día de la semana, éste fue el grado de opción disponible para el consumidor en México a una hora determinada durante 1999. La informa-

6. El horario A va de las 6:00 a las 14:59 y de 23:01 a 23:59 -para fines de este estudio-, el AA de las 15:00 a las 18:59 y el AAA de 19 a 23 horas. 
ción, que en el plano general observó una alta concentración, pierde su importancia a lo largo de un día común, y aunque su presencia sigue siendo alta en el horario Triple A (10\%), no llega a superar a la ficción. Es preocupante que precisamente en el horario de mayor audiencia la concentración sea mayor y por tanto el grado de oportunidad de la audiencia menor. Los datos permiten señalar, como ya se dijo, que la diversidad horizontal es baja en todos los casos.

Aunque en el horario AAA no hay mayor concentración que en el resto, sí parece haber menos concentración en el horario de menor audiencia. Los datos sugieren que existió escasa diversidad vertical tanto como horizontal, por lo que parece posible decir que la programación de la televisión abierta nacional durante 1999 fue poco diversa. La concentración de la programación abierta mexicana durante 1999 dificulta hablar de diversidad externa al sistema. ${ }^{7}$ Ésta se hubiera dado si a pesar de ser escasa la diversidad en la estructura televisiva, la diversidad programática hubiera ocurrido, lo que parece coincidir con las posturas de Kim (1996) e Ishikawa (1996), respecto a la estrecha relación de la estructura del sistema con su oferta programática.

\section{Diversidad cultural}

La procedencia de la programación importada es, de nueva cuenta, poco diversa. Las preocupaciones respecto a la alta cantidad de importaciones se han sofisticado, desde que se ha mostrado que países productores de programación tienden a programar una mayor cantidad de televisión local que aquellos que no lo son (Norris y Pauling, 1999). Siendo México uno de los mayores productores de programas de televisión en el mundo, y una de sus empresas - Televisa- la mayor productora de programas en castellano, es esperable que su programación local sea más alta que la importada, aunque no tanto, si tomamos en cuenta la cantidad tradicionalmente fluctuante (Lozano, 1997). Los datos acerca de la procedencia de la programación fueron consistentes a lo largo de 1999, como se puede observar en la tabla 5.

Este trabajo coincide con Vincent (1997) y en un plano más general, con Boyer (1997), en el sentido de que los fenómenos sociales, particularmente los culturales, en plena era de la globalidad siguen teniendo un principio local, y la televisión no es la excepción. Lo que resulta preocupante es la escasa diversidad en materia de importaciones, pero de esto se hablará más tarde. Por ahora baste mostrar que el origen de las importaciones es en casi todos los casos Estados Unidos de América. Aun con la suma de los valores perdidos, donde en ocasiones se introdujo algún

7. Como se muestra en otro lado (Huerta Wong, 2000a y 2000b), no parece posible hablar de diversidad interna dada la concentración de la propiedad de las estaciones televisoras locales y nacionales, que detentan casi absolutamente dos empresas. 
programa de otra procedencia, las importaciones de Japón (3.8\%), Colombia (.7\%), Venezuela (.1\%) y España (.8\%) no rebasan el 25 por ciento del total de la programación importada. Contrario a lo que se esperaba, la presencia de la programación importada no crece en el horario Triple A, y aunque la ficción estadounidense es alta en ese horario (48\%), nunca es mayor a la programación local, como se muestra en el Cuadro 6. Estos mismos datos, como los resultados antes mostrados, observan una radicalización de la programación importada. Las lecciones de la televisión, por lo menos según su programación, son que toda la realidad que no está en México, se encuentra en Estados Unidos de América. Algo de eso se discutirá más adelante.

Las importaciones, como se ha observado en otras regiones del mundo (Hultén, 1995; Ischii, Su y Watanabe, 1999), parecen estar especializadas. Los programas españoles sólo son de variedades y entretenimiento $(3 \%$, respecto del total de programación en ese rubro) o infantiles (2\%, id.); Estados Unidos de América tiene una importante participación en deportes, particularmente en horario Triple A $(28 \%$, id); mientras que todos los programas que se importan de Japón son infantiles y de Colombia de ficción. En general, los datos fueron un poco sorprendentes. No sólo es la inusual concentración de la programación local (que estudios como Lozano, 1997, entre otros, han mostrado que suele ser más baja), sino también la inusual cantidad de ficción estadounidense (que suele ser más alta). De acuerdo con estos datos, podemos hablar de que la alta concentración de la programación abierta mexicana también conlleva un crecimiento en la concentración de la programación local en los formatos que aglutinan la mayor cantidad de oferta disponible, información y ficción. Hay en esto implicaciones que discutir más adelante. Parece haber suficiente evidencia para respaldar la afirmación de que la televisión abierta nacional hizo ligero énfasis en la oferta de la programación local, con escasa diversidad en la programación importada.

\section{Discusión}

Se ha propuesto que un principio de evaluación de la televisión -en este caso la mexicana-es la observación de la diversidad y se estableció que un punto de partida debía ser la estructura y la oferta programática, para lo cual se distinguió el concepto de diversidad en la programación del de variedad, que se refiere al contenido de los programas y no a los programas como unidades narrativas.

Se establecieron dos preguntas de investigación, ¿Qué tanta diversidad encontramos en el sistema de canales abiertos mexicanos? y icuáles son los tipos televisivos más difundidos y qué tan equilibrada se encuentra la oferta de los mismos?

Los hallazgos de este trabajo permiten contestar así las preguntas anteriores: la diversidad en el sistema de canales abiertos mexicanos es escasa, la programa- 
ción de la televisión abierta nacional durante 1999 fue poco diversa y la televisión abierta nacional presentó un ligero énfasis en la oferta de programación local, con escasa diversidad en la programación importada. Por otro lado, el grado de diversidad presente en la programación de la televisión abierta nacional durante 1999 tendió a parecerse a la existente en el país o región de donde proviene la mayor cantidad de programación importada, y el cumplimiento de la responsabilidad de la televisión abierta mexicana en 1999, de acuerdo con el ideal de servicio público deseable en una comunidad democrática, fue insatisfactorio.

\section{Implicaciones}

A lo largo de este trabajo se han planteado varios puntos. El primero de ellos tiene que ver con revisar el concepto de la responsabilidad (en el sentido de rendición de cuentas) de la televisión en particular y en general del sistema de medios de comunicación masiva en la transición democrática de México. Se ha propuesto que esta responsabilidad tiene que ver con la satisfacción de intereses de consumo mediático de todos los grupos sociales. Eso es problemático porque, como admiten Ishikawa y Muramatzu (1996), podría por otro lado suponerse que en realidad el sistema de medios de comunicación no cambia porque es el último indicador de que la cultura está modificándose, como ha dicho Borrat (1989), y en realidad no es exigido para que ese cambio se dé. La evidencia respecto a la discrepancia entre grado de satisfacción y consumo (Legatt, 1996) permite ser escéptico con eso, pero por otra parte, no parece haber datos en México del grado de satisfacción de las audiencias.

Otro punto problemático es la definición de calidad a partir del cumplimiento -o no- de esta responsabilidad. Nuevamente aquí hacen falta datos de qué entenderían las audiencias como calidad y cuál debería ser según los consumidores, el rol de la televisión en específico, y de los medios de comunicación en general.

Pero si concedemos alguna validez a estos principios, entonces la transición democrática de México adolece de un prerrequisito fundamental. La televisión, al concentrar su oferta programática en unas cuantas fórmulas, está siendo excluyente.

Para que pueda cumplirse con el ideal de responsabilidad, es necesario que existan tres elementos: medios de comunicación, quien pide la responsabilidad (sociedad civil demandante) y un árbitro; cuatro dimensiones: temas y principios normativos, formas y discurso, procedimientos y grado de libertad; tres marcos prevalecientes: un marco legal-regulatorio, un marco de mercado financiero, un marco de confianza pública.

En México casi todo esto falta. Hay pocos medios de comunicación, prácticamente no existe quién pida responsabilidad (cfr. Corral, 1998) y tampoco hay un árbitro (Carreño Carlón y Villanueva, 1998); la libertad conseguida por los medios 
actúa excluyendo a las audiencias (Corral, 1998); no existe un marco legalregulatorio (Carreño Carlón y Villanueva, 1998) y la confianza pública hacia casi cualquier institución de mediación es nula (Ramos Gómez y Durán Ponte, 1997).

Luego, el cumplimiento de esta responsabilidad no puede darse de manera lineal, sino que deben participar todos los actores involucrados con la producción, oferta, distribución y consumo de las formas mediáticas. La televisión no contribuye a la construcción del interés público en México, o no lo hizo al menos en 1999 desde esta particular visión, y no parecen existir condiciones estructurales en las diferentes fases del proceso comunicativo como para que esto cambie. ¿Qué tipo de estructura del sistema televisivo garantiza la diversidad? Este trabajo no da esa respuesta, pero sí se puede entender que no es la que tiene el sistema televisivo mexicano.

Ahora bien, esto tampoco quiere decir que: a) no se pueda cambiar esta realidad y b) la transición mexicana no pueda darse sin medios inclusivos.

Villanueva (1998a) ha dicho que la diversidad y pluralidad de las cartas televisivas es el sello de un estado democrático de derecho, pero él mismo no incluyó este tema en la ley de comunicación social que redactó (Villanueva, 1998b), y cada vez son más voces las que claman por una reglamentación de los artículos 6 y 7 constitucionales.

Asimismo, la transición mexicana ha sido lenta y muy probablemente lo seguirá siendo, de modo que se puede ser optimista y pensar que, tarde que temprano, los cambios sociales van a incorporar virajes en la toma de decisiones de los medios de comunicación. En tanto, es necesario contribuir en alguna medida para que la reglamentación, como punto de partida al cumplimiento de la responsabilidad social de los medios, pueda darse. Este trabajo es una forma de haccrlo, pero es necesario el esfuerzo de expertos en políticas públicas, para la justificación y redacción de una ley a la medida de la transición. En el caso de la televisión, quizá sólo políticas que acoten los monopolios e impulsen a la televisión de servicio público así sea sólo mejorando los tiempos de programas públicos al interior de las televisoras comerciales- pueda mejorar el grado de diversidad existente.

La televisión es el medio de transmisión de formas simbólicas más importante en México pero no es el único. Los medios de comunicaciones ${ }^{8}$ han tenido un continuo ascenso, pero como están las cosas -y a pesar de sus características-, se puede especular que la realidad no sea muy distinta ahí. Hay que considerar, empero, que las audiencias tienen mucho mayor margen de acción en algunos de estos nuevos medios, lo que ha despertado una discusión respecto a sus potencialidades democráticas. Hace faltan datos que respalden cualquiera de las partes críticas o entusiastas- de esta discusión.

8. Straubhaar y LaRose (1997) han acuñado el término "medios de comunicaciones" para referirse a la convergencia de medios masivos tradicionales, computadoras y satélites. 
Cuadro 1. Tipología de formatos

\begin{tabular}{|c|c|}
\hline Formato & Tipo \\
\hline Información & $\begin{array}{l}\text { Noticiero } \\
\text { Entrevista y debate } \\
\text { Magazine (noticias, entrevistas y música) } \\
\text { Comentarios humorísticos }\end{array}$ \\
\hline Ficción & $\begin{array}{l}\text { Película (cine) } \\
\text { Miniserie } \\
\text { Series: de comedia (Comedia de situación), de aventura o ficción, de } \\
\text { drama/teleteatro, de suspenso, de ciencia ficción, de terror o sobrenatural, } \\
\text { policiaca } \\
\text { Cómicos } \\
\text { Telenovelas }\end{array}$ \\
\hline Infoshow & $\begin{array}{l}\text { Tele-verdad } \\
\text { Talk show/Chat } \\
\text { Pseudo-ciencia } \\
\text { Videos de aficionados }\end{array}$ \\
\hline $\begin{array}{l}\text { Variedades y } \\
\text { entretenimiento }\end{array}$ & $\begin{array}{l}\text { Juegos y concursos } \\
\text { Musicales } \\
\text { Cocina } \\
\text { Humorísticos } \\
\text { Crónica social } \\
\text { Asistencia social } \\
\text { Reseñas, cortos y "makings" de películas }\end{array}$ \\
\hline Infantiles & $\begin{array}{l}\text { Actividades y concursos } \\
\text { Dibujos animados } \\
\text { Series infantiles } \\
\text { Musicales } \\
\text { Didácticos } \\
\end{array}$ \\
\hline Deportes & Comentarios, análisis, entrevistas \\
\hline Culturales & $\begin{array}{l}\text { Documentales } \\
\text { Concursos educativos } \\
\text { Reportajes }\end{array}$ \\
\hline Educativos & Cursos de educación formal \\
\hline Telemercadeo & Programas de ventas directas al público \\
\hline Propaganda politica & $\begin{array}{l}\text { Propaganda política pagada } \\
\text { Partidos políticos (tiempo oficial) } \\
\text { Acto civico } \\
\text { Mensaje a la nación }\end{array}$ \\
\hline
\end{tabular}




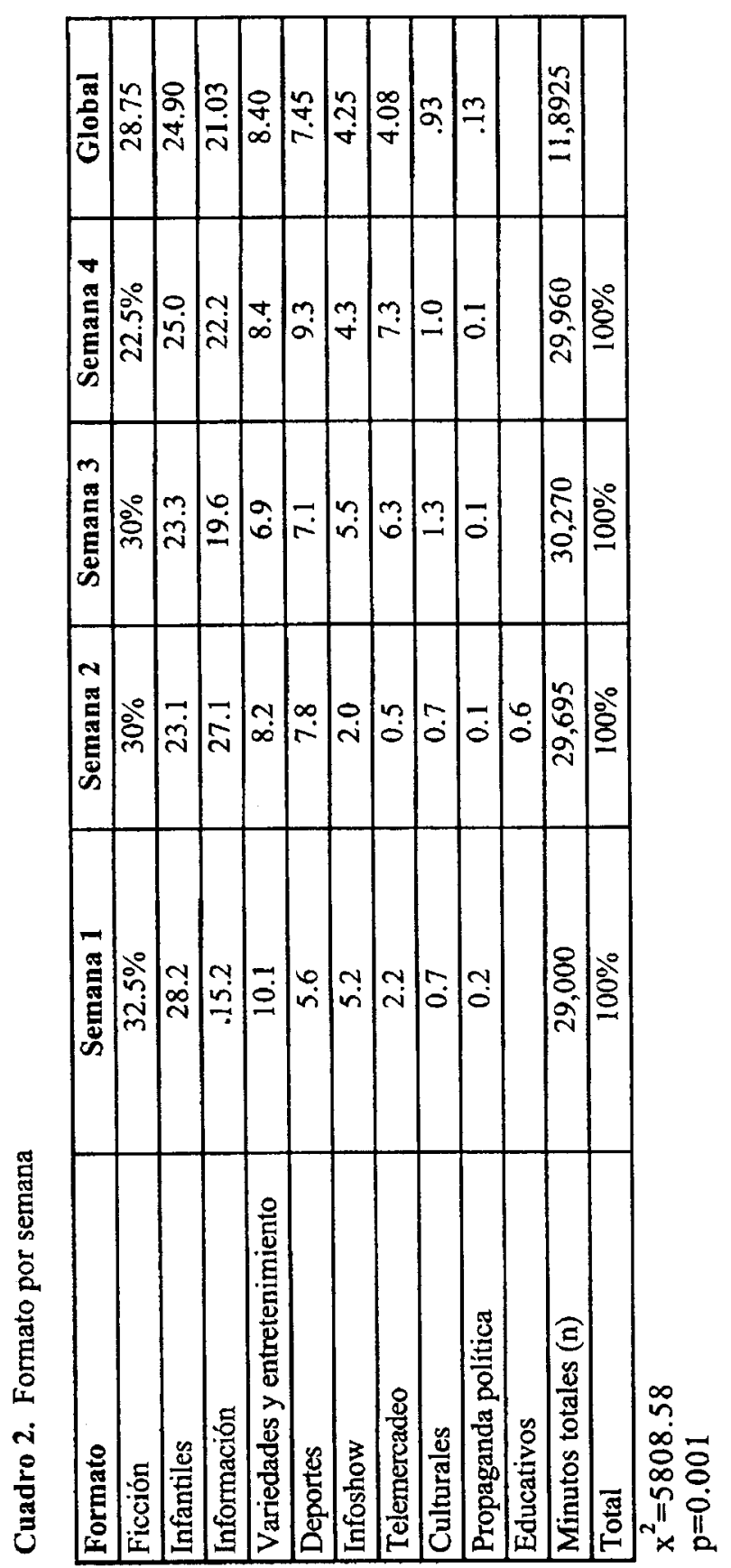




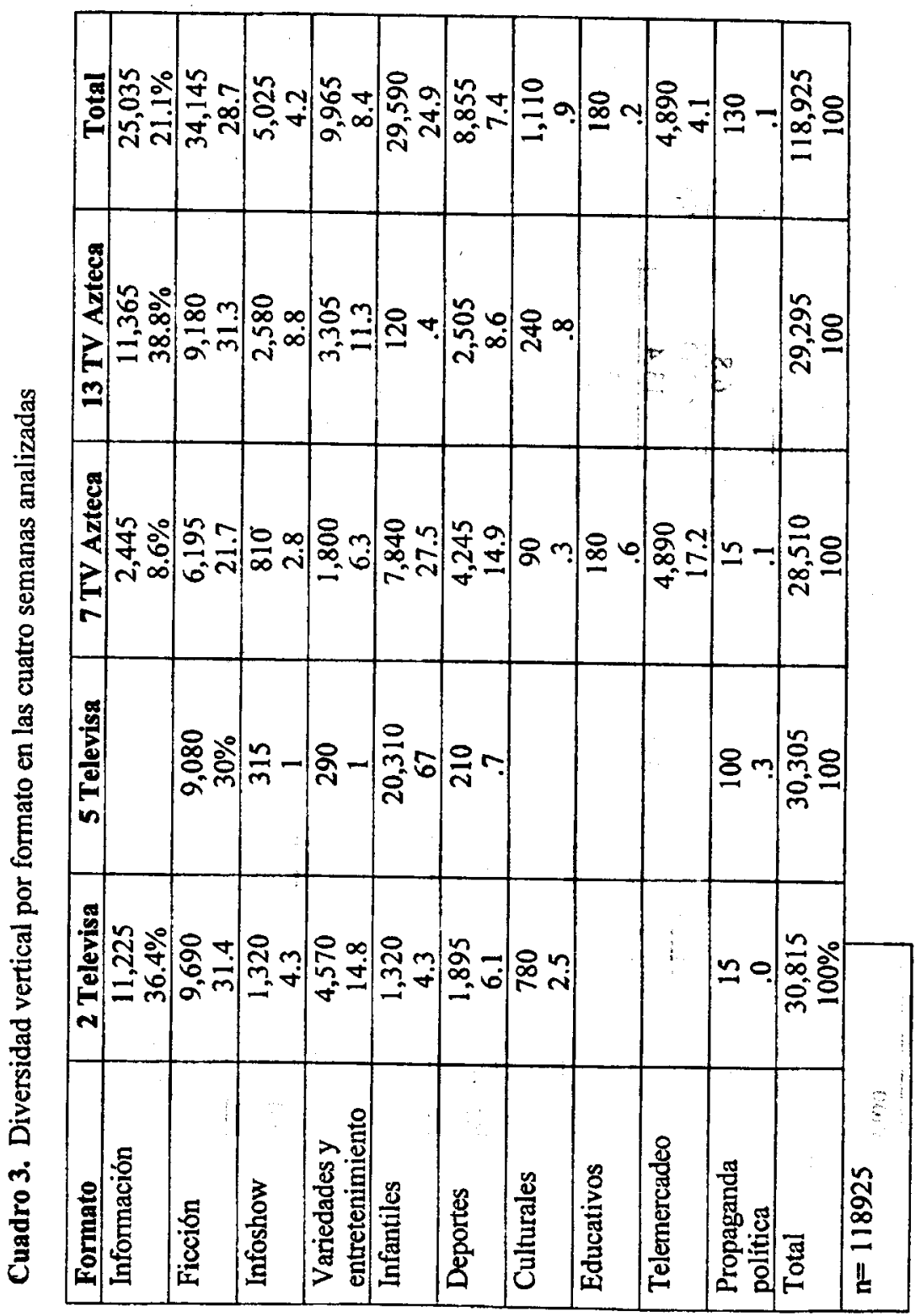




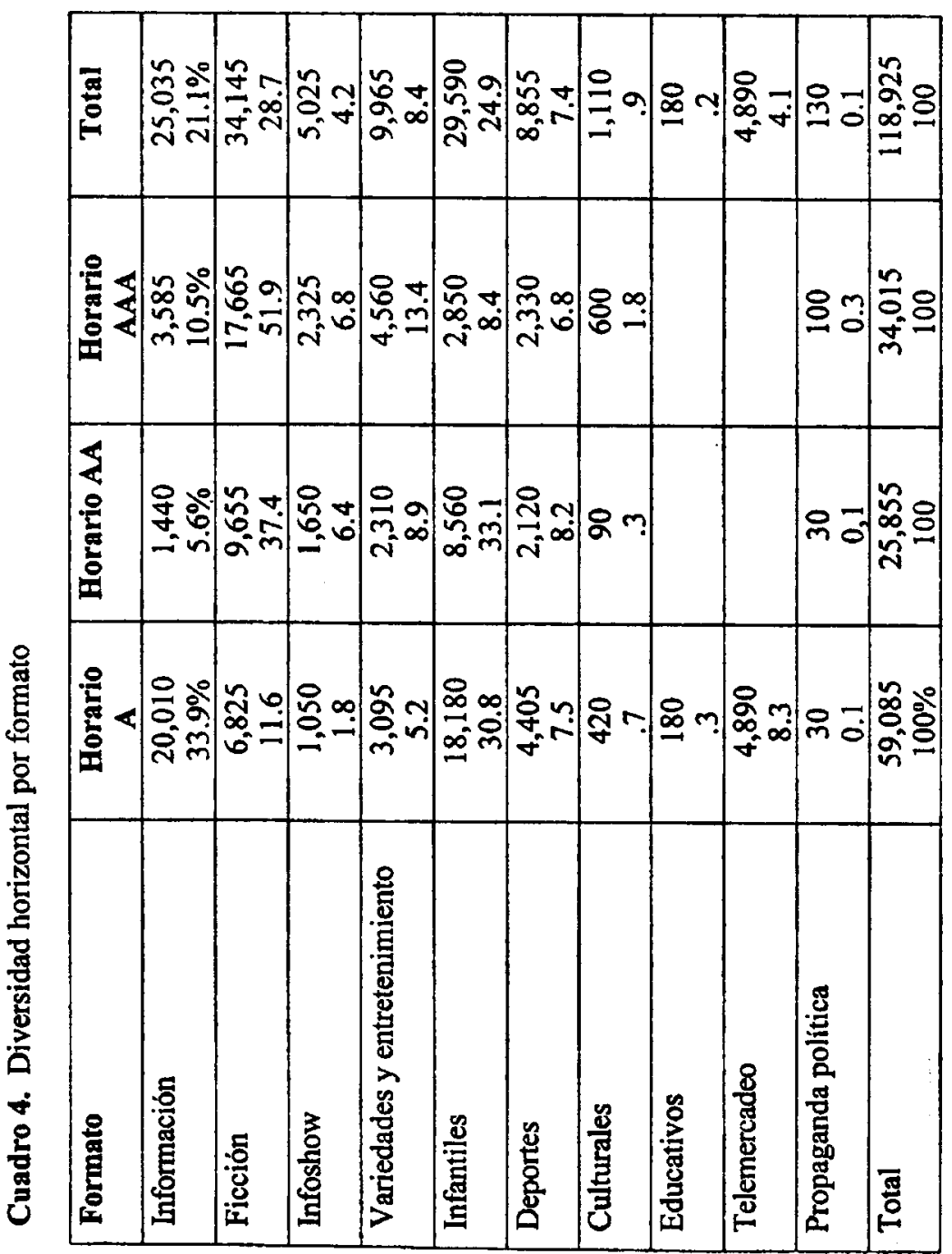




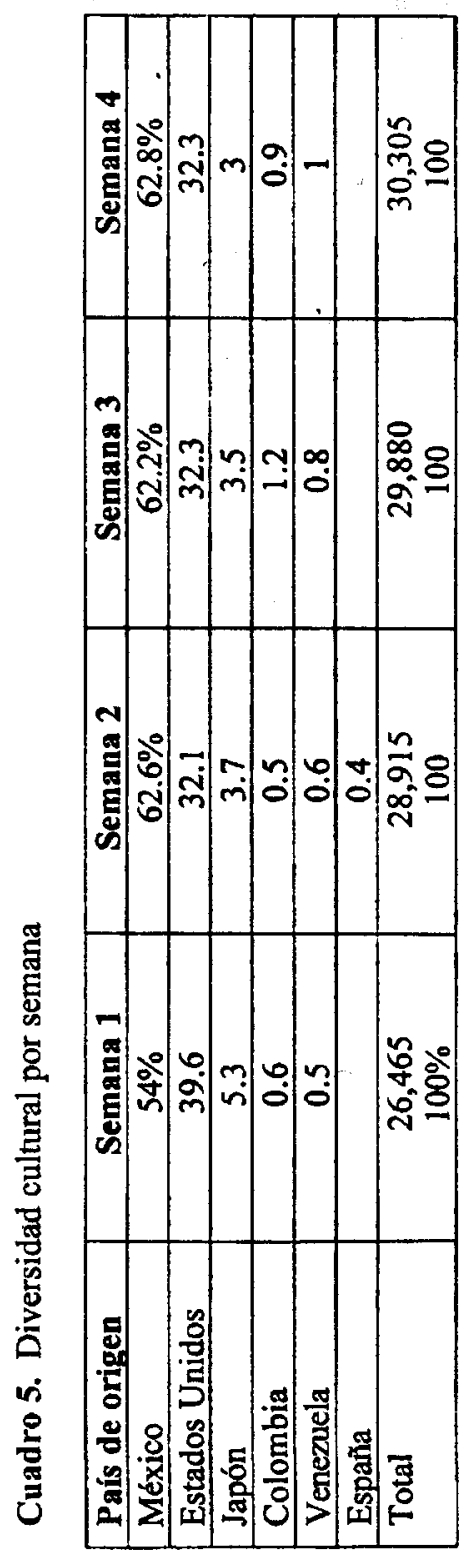


Cuadro 6. Diversidad cultural, desglose por formatos

\begin{tabular}{|c|c|c|c|c|c|}
\hline Formato & País de origen & $\begin{array}{c}\text { Horario } \\
\mathbf{A}\end{array}$ & $\begin{array}{c}\text { Horario } \\
\text { AA }\end{array}$ & $\begin{array}{c}\text { Horario } \\
\text { AAA }\end{array}$ & Total \\
\hline \multirow[t]{3}{*}{ Información } & México & $100 \%$ & $100 \%$ & $100 \%$ & $100 \%$ \\
\hline & Otros paises & 0 & 0 & 0 & 0 \\
\hline & $\begin{array}{l}\text { Total (n) } \\
\quad \text { Porcentaje horario }\end{array}$ & $100 \%$ & $100 \%$ & $100 \%$ & $\begin{array}{l}25035 \\
100 \% \\
\end{array}$ \\
\hline \multirow[t]{4}{*}{ Ficción } & México & 52.7 & 57.8 & 48.4 & $51.8 \%$ \\
\hline & Estados Unidos & 47.3 & 38.9 & 48.4 & 45.5 \\
\hline & Colombia & 0 & 3.3 & 3.2 & 2.7 \\
\hline & $\begin{array}{l}\text { Total (n) } \\
\text { Porcentaje } \\
\end{array}$ & $100 \%$ & $100 \%$ & 100 & $\begin{array}{c}31340 \\
100\end{array}$ \\
\hline \multirow[t]{3}{*}{ Infoshow } & México & 77.1 & 98.1 & 76.8 & 83.8 \\
\hline & Estados Unidos & 22.9 & 1.9 & 23.2 & 16.2 \\
\hline & $\begin{array}{l}\text { Total (n) } \\
\quad \text { Porcentaje horario }\end{array}$ & 100 & 100 & 100 & $\begin{array}{l}4995 \\
100\end{array}$ \\
\hline \multirow[t]{4}{*}{$\begin{array}{l}\text { Variedades y } \\
\text { entretenimiento }\end{array}$} & México & 70.3 & 91.3 & 92.8 & 85.3 \\
\hline & Estados Unidos & 19.1 & 8.7 & 7.2 & 11.3 \\
\hline & España & 10.7 & 0 & 0 & 3.4 \\
\hline & $\begin{array}{l}\text { Total (n) } \\
\quad \text { Porcentaje horario }\end{array}$ & 100 & 100 & 100 & $\begin{array}{c}9725 \\
100 \\
\end{array}$ \\
\hline \multirow[t]{6}{*}{ Infantiles } & México & 10.6 & 12.4 & 0 & 10.1 \\
\hline & Estados Unidos & 71.3 & 82.7 & 43.2 & 71.9 \\
\hline & Japón & 13.9 & 4.9 & 56.8 & 15.5 \\
\hline & Venezuela & 0.7 & 0 & 0 & 0.4 \\
\hline & España & 3.5 & 0 & 0 & 2.1 \\
\hline & $\begin{array}{l}\text { Total (n) } \\
\quad \text { Porcentaje horario }\end{array}$ & 100 & 100 & 100 & $\begin{array}{c}28630 \\
100\end{array}$ \\
\hline \multirow[t]{3}{*}{ Deportes } & México & 84.6 & 85 & 71.7 & 81.2 \\
\hline & Estados Unidos de América & 15.4 & 15 & 28.3 & 18.8 \\
\hline & $\begin{array}{l}\text { Total (n) } \\
\text { Porcentaje horario }\end{array}$ & 100 & 100 & 100 & $\begin{array}{c}8615 \\
100 \\
\end{array}$ \\
\hline \multirow[t]{3}{*}{ Culturales } & Mexico & 100 & 100 & 95 & 97.3 \\
\hline & Estados Unidos & 0 & 0 & 5 & 2.7 \\
\hline & $\begin{array}{l}\text { Total (n) } \\
\text { Porcentaje horario }\end{array}$ & 100 & 100 & 100 & $\begin{array}{c}1110 \\
100\end{array}$ \\
\hline \multirow[t]{3}{*}{ Educativos } & Estados Unidos & 100 & 100 & 100 & 100 \\
\hline & México & 0 & 0 & 0 & 0 \\
\hline & $\begin{array}{l}\text { Total (n) } \\
\quad \text { Porcentaje horario }\end{array}$ & 100 & 100 & 100 & $\begin{array}{l}180 \\
100\end{array}$ \\
\hline \multirow[t]{3}{*}{ Telemercadeo } & México & 87.1 & 0 & 0 & 87.1 \\
\hline & Estados Unidos & 12.9 & 0 & 0 & 12.9 \\
\hline & $\begin{array}{l}\text { Total (n) } \\
\text { Porcentaje horario }\end{array}$ & 100 & 0 & 0 & $\begin{array}{l}4260 \\
100\end{array}$ \\
\hline \multirow[t]{3}{*}{$\begin{array}{l}\text { Propaganda } \\
\text { política } \\
\end{array}$} & México & 100 & 0 & 100 & 100 \\
\hline & Otros paises & 0 & 0 & 0 & 0 \\
\hline & $\begin{array}{l}\text { Total (n) } \\
\text { Porcentaje horario }\end{array}$ & 100 & 0 & 100 & $\begin{array}{l}130 \\
100\end{array}$ \\
\hline
\end{tabular}


La concentración de la oferta programática es todavía más alta para los públicos que necesitarían una mayor diversidad, los infantiles. El no reflejar los intereses de una gran parte de esta población, podría representar que el tiempo en que los actores sociales tarden en saltar el escollo de la diversidad televisiva demore aún más. Esto repercutiría negativamente en el acceso a la dimensión comunicativa de la democracia, con algún costo en la transición.

\section{Limitaciones}

Las limitaciones de este trabajo son altas, debido a factores como la dificultad de definir categorías como responsabilidad (o rendición de cuentas), calidad, diversidad, programación; otras limitaciones tienen que ver con el alcance de los métodos cuantitativos.

Se hizo constante la presencia de la limitación de la tipología. Una tipología no orientada a la oferta existente sino a la deseable, puede ser mejor en futuros estudios. También se pueden trabajar réplicas a los resultados de Kim (1996) o de Ishikawa et al. (1996), los más grandes trabajos que parecen haberse realizado hasta este momento. Los mismos datos pueden volver a ser revisados para definir con mayor transparencia el grado de diversidad y las correlaciones existentes entre la oferta y la programación y entre la diversidad de la programación y la diversidad cultural de la misma.

Más trabajo puede ser realizado revisando la variedad al interior de los programas y sus significados semióticos. También pueden ser revisados las demás fases en el proceso comunicativo, preguntando a las audiencias y a productores qué entienden por calidad y/o responsabilidad de la televisión en este inicio de siglo en México.. Se puede revisar más a detalle la diversidad en los sistemas públicos, regionales y privados televisivos, a fin de contrastar los hallazgos aquí mostrados, tanto en la parte de la oferta, como de la diversidad programática y cultural.

\section{Conclusión}

El presente trabajo planteó preguntas acerca de la diversidad existente en la programación de la televisión abierta mexicana, con el objetivo de revisar su rol desde el punto de vista de la responsabilidad como grado de oportunidad del sistema televisivo a la satisfacción de intereses de todos los actores sociales. El supuesto fue que un sistema social incluyente produce un sistema televisivo también incluyente, ${ }^{9}$ al cual le llamamos de calidad. Su característica es la diversidad.

9. Parte importante del sistema televisivo, la estructura, no es analizada en este trabajo por razones de espacio. El análisis al respecto, complemento de este texto, puede encontrarse en otros trabajos (Huerta Wong, 2000a y 2000b). 
Nuestro diagnóstico del sistema televisivo mexicano es que en 1999 se comportó de manera excluyente y por tanto incumplió su responsabilidad social. Como ya se mencionó, este trabajo no intentaba dar soluciones sino evaluar y diagnosticar. Pero una cosa debe ser apuntada. Si bien el tema de este artículo no es cómo acercarse a una mejor televisión, si podemos decir qué entendemos por "mejor" televisión. Desde nuestro punto de vista, con base en la discusión internacional acerca de la calidad en la televisión, un sistema televisivo debe garantizar oportunidad de satisfacción de los intereses de la mayor parte posible de su audiencia y esto sólo parece posible aumentando el grado de diversidad. Como se ha revisado, cada país ha encontrado -o por lo menos buscado- soluciones diferentes a este problema, del cual la reglamentación es un primer paso. Se ha propuesto también que la reglamentación podría incluir el fortalecimiento de programas públicos en la televisión comercial, cuya naturaleza no parece posible de modificar, por lo menos no en el corto plazo. Ésa no es la única forma. Aumentar la competencia existente en las prácticas monopólicas del mercado televisivo mexicano podría ser otra manera, considerando que la estructura del sistema aparece estrechamente ligada a su programación.

\section{Referencias}

Biltereyst, D. (1992). Language and culture as ultimate barriers?: An analysis of the circulation, consumption and popularity of fiction in small European countries, en European Journal of Communication, núm. 7, pp. 517-540.

Borrat, H. (1989). El periódico, actor político. Barcelona, G. Gili.

Boyer, R. (1997). La globalización: mitos y realidades, en E. Gutiérrez Garza, (Coord.), El debate nacional (vol. 1, pp. 21-42). México, Editorial Diana-Universidad Autónoma de Nuevo León.

Carreño Carlón, J. y Villanueva, E. (1998). Derecho de la información en México, en J. Carreño Carlón y E. Villanueva (eds.), Temas fundamentales de derecho de la información en Iberoamérica (pp. 141-158). Madrid: Fragua Editorial/ México: Universidad Iberoamericana.

Corral Jurado, J. (1998). Derecho a la información, en Comisión de Radio, Televisión y Cinematografía de la $\mathrm{H}$. Cámara de Diputados, La reforma de medios: Voces en torno a la iniciativa de Ley Federal de Comunicación Social (pp. 21-28). México, Autor.

Crovi Druetta, D. y Vilar Alcalde, J. (1995). Canales abiertos de la Ciudad de México: Programación y preferencias del público, en D. Crovi Druetta (Ed.), Desarrollo de las industrias audiovisuales en México y Canadá (pp. 177-192). México, Facultad de Ciencias Políticas y Sociales de la Universidad Nacional Autónoma de México. 
De Bens, E. (1998). Television programming: more diversity, more convergence?, en K. Brants, J. Hermes y L. van Zoonen (eds.), The media in question: popular cultures and public interests (pp. 27-37). Londres: Sage Publications.

De la Garde, Roger (1993). Dare we compare?, en R. de la Garde, W. Gilsdorf e I. Wechselmann (Eds.), Small nations, big neighbour: Denmark and Quebec/ Canada compare notes on american popular culture (pp. 25-64). Londres, John Libbey \& Company LTD.

Fuentes Navarro, R. (1988). La investigación de la comunicación en México: Sistematización documental 1986-1996. Guadalajara, Instituto Tecnológico y de Estudios Superiores de Occidente-Universidad de Guadalajara.

- (1996). La investigación de la comunicación en México: Sistematización documental 1956-1986. México, Ediciones de Comunicación.

Gates, Arlan (1998). What's on TV? An analysis of the availability of local/regional and Canadian programming in Winnipeg in 1997/98 (with comparative data from Vancouver). Canadá, Friends of Canadian Broadcasting. Disponible en: friendsb.org/research/1998-44whatsontv.htm.

Golding, P. (1998). New technologies and old problems: evaluating and regulating media performance in the "information age", en K. Brants, J. Hermes y L. van Zoonen (eds.), The media in question: popular cultures and public interests (pp. 7-17). Londres, Sage Publications.

Gutiérrez, C. (2000). Televisión y calidad: perspectivas de investigación y criterios de evaluación, en ZER Revista de Estudios de Comunicación, (9). Disponible en: http://www.ehu.es/zer/

Hillve, P. (1995). After research, measurement or perhaps Vice Versa?, en The Nordicom Review, núm. 1, pp. 23-36.

- Majanen, P. y Rosengren, K. E. (1997). Aspects of quality in TV programming: Structural diversity compared over time and space, en European Journal of Communication, núm. 12 (3), pp. 291-318.

Huerta Wong, J. E. (2000). Hacia una evaluación de la televisión mexicana: diversidad en la programación durante 1999. Tesis de Maestría en Ciencias con especialidad en Comunicación, Tecnológico de Monterrey.

Hultén, O. (1995). Diversity or conformity? Television programming in competitive situations, en The Nordicom Review, núm. 1, pp. 7-21.

Ischii, K.; Su, H. y Watanabe, S. (1999). Japanese and US programs in Taiwan: New patterns in Taiwanese television, en Journal of broadcasting \& electronic media, núm. 43 (3),pp. 416-431.

Ishikawa, S. y Muramatsu, Y. (1996). Why measure diversity? , en S. Ishikawa (Ed.), Quality Assessment of Television (pp. 199-202). Gran Bretaña, University of Luton Press.

Ishikawa, S.; Leggatt, T; Litman, B.; Raboy, M.; Rosengren, K. E. y Kambara, N. (1996). Diversity in television programming: Comparative analysis of five 
countries, en S. Ishikawa (Ed.), Quality Assessment of Television (pp. 253-264). Gran Bretaña, University of Luton Press.

Ishikawa, Sakae (ed.) (1996). Quality Assessment of Television. Gran Bretaña: University of Luton Press.

Kim, D. (1996). Television program diversity and media structure: $A$ cross national comparative study. Tesis doctoral, Universidad de Texas en Austin.

Lerche-Nielsen, Joergen e Ilja Wechselmann (1993). Does internationalization impose a crisis on Denmark?, en R. De la Garde, W. Gilsdorf e I. Wechselmann (eds.), Small nations, big neighbour: Denmark and Quebec/Canada compare notes on american popular culture, (pp. 103-120). Londres, John Libbey \& Company LTD.

Lozano, J. C. (1995/96). Oferta y recepción de televisión extranjera en México, en Comunicación y Sociedad, 25-26, 259-284.

- (1997, julio). Oferta y consumo de contenidos televisivos transnacionales en México. Ponencia presentada en la XL Conferencia Anual de la Asociación Internacional de Estudios de Comunicación Social, Oaxaca.

- (1998). Oferta de programas televisivos extranjeros en el área metropolitana, en M. Ceballos Ramírez (coord.), Monterrey 400: Estudios históricos y sociales (pp. 301-320). Monterrey, Universidad Autónoma de Nuevo León.

Lozano, J. C. y García Núñez de Cáceres, J. (1995). Oferta de programación audiovisual extranjera en la televisión aérea de Monterrey, Nuevo León, en D. Crovi Druetta, Desarrollo de las industrias audiovisuales en México y Canadá (pp. 215-230). México, Facultad de Ciencias Políticas y Sociales de la Universidad Nacional Autónoma de México.

McQuail, D. (1997). Accountability of media to society, en European Journal of Communication, núm. 12 (4), pp. 511-529.

- (1998). La acción de los medios: Los medios de comunicación y el interés público. Buenos Aires, Amorrortu editores.

Norris, P.y Pauling, B. (1999). Local content and diversity: television in ten countries. Nueva Zelanda: NZ on air. Disponible en: www.nzonair.govt.nz/ local content $99 /$ exec/index.html

Ramos Gómez, F. y Durán Ponte, V. M. (1997, agosto). Los universitarios y la confianza en las instituciones, en Este país, 10-13.

Rosengren, K. E.; Carlsson, M.; y Tagerud, Y. (1996). Quality in programming: Views from the North, en S. Ishikawa (Ed.), Quality Assessment of Television (pp. 3-48). Gran Bretaña: University of Luton Press.

Sánchez Ruiz, E. (1996). Flujos globales, nacionales y regionales de programación televisiva: El caso de México, en Comunicación y Sociedad, núm. 27, pp. 43-88.

- (1992). Tendencias en la investigación sobre comunicación en México: 1950-1990. Guadalajara, CEIC-Universidad de Guadalajara.

- (1995). La agenda televisiva en Guadalajara, en D. Crovi Druetta (Ed.), De- 
sarrollo de las industrias audiovisuales en México y Canadá, 193-213. México, Facultad de Ciencias Políticas y Sociales de la Universidad Nacional Autónoma de México.

Straubhaar, J. y LaRose, R. (1996). Communications media in the information society. Belmont, Cal., Wadsworth Pub.

The new policy on Canadian television: More flexibility, diversity and programming choice (2000, febrero). Disponible en: www.newswire.ca/government/federal/ english/releases/June1999/11/c3361.html

Thompson, J. B. (1993). Ideología y cultura moderna: teoría crítica y social en la era de la comunicación de masas. México, Universidad Autónoma Metropolitana. - (1998). Los media y la modernidad: Una teoría de los medios de comunicación. Paidós Comunicación: Barcelona.

Van Cuilenberg, J. (1998). Diversity revisited: towards a critical rational model of media diversity, en K. Brants, J. Hermes y L. van Zoonen (Eds.), The media in question: popular cultures and public interests (pp. 38-49). Londres, Sage Publications. Tremblay, G. y Gauvreau, C. (1995). La oferta televisiva francófona y su consumo en Montreal, en D. Crovi Druetta (Ed.), Desarrollo de las industrias audiovisuales en México y Canadá (pp. 161-176). México: Facultad de Ciencias Políticas y Sociales de la Universidad Nacional Autónoma de México.

Varis, T. (1988). Trends in international television flow, en C. Schneider y B. Wallis (eds.), Global television (pp. 95-107). EE.UU., The MIT Press/Wedge Press. Villanueva, E. (1998a). Derecho comparado de la información. México, Universidad Iberoamericana.

- (1998b). Régimen jurídico de las libertades de expresión e información en México. México: Universidad Nacional Autónoma de México.

Vincent, R. C. (1997). The future of the debate: Setting an agenda for a New World Information and Communication Order, ten proposals, en P. Golding y P. Harris (eds.), Beyond cultural imperialism: Globalization, communication and the new international order. London, Thousand Oaks. 